\title{
Space-Time Cluster Analysis of Tuberculosis Incidence in Beijing, China
}

\author{
Gehendra Mahara1,2,3, Mina Karki4, Kun Yang1,3,5, Sipeng Chen 1,3,6, Wei Wang1,7*, Xiuhua Guo ${ }^{1,3^{*}}$ \\ ${ }^{1}$ Department of Epidemiology and Health Statistics, School of Public Health, Capital Medical University, Beijing, China \\ ${ }^{2}$ Department of Medical Statistics and Epidemiology, School of Public Health, Sun Yat-sen University, Guangzhou, China \\ ${ }^{3}$ Beijing Municipal Key Laboratory of Clinical Epidemiology, Beijing, China \\ ${ }^{4}$ Mental Hospital, Kathmandu, Nepal \\ ${ }^{5}$ Department of Evidence-Based Medicine, Xuanwu Hospital, Beijing, China \\ ${ }^{6}$ Fuwai Hospital, Chinese Academy of Medical Science, Beijing, China \\ ${ }^{7}$ School of Medical Sciences, Edith Cowan University, Perth, Australia \\ Email: gbmahara@gmail.com, karkimina33@hotmail.com, yangkun1123@163.com, ivanchen2010sp@hotmail.com, \\ *wei.wang@ecu.edu.ac, *guoxiuh@ccmu.edu.cn
}

How to cite this paper: Mahara, G., Karki, M., Yang, K., Chen, S.P., Wang, W. and Guo, X.H. (2018) Space-Time Cluster Analysis of Tuberculosis Incidence in Beijing, China. Journal of Tuberculosis Research, 6, 302-319.

https://doi.org/10.4236/jtr.2018.64027

Received: December 6, 2018

Accepted: December 25, 2018

Published: December 28, 2018

Copyright (c) 2018 by authors and Scientific Research Publishing Inc. This work is licensed under the Creative Commons Attribution International License (CC BY 4.0)

http://creativecommons.org/licenses/by/4.0/

\begin{abstract}
Tuberculosis is one of the top killer diseases in the globe. The aim of this study was to explore the geographic distribution patterns and clustering characteristics of the disease incidence in terms of both space and time with high relative risk locations for tuberculosis incidence in Beijing area. A retrospective space-time clustering analysis was conducted at the districts level in Beijing area based on reported cases of sputum smear-positive pulmonary tuberculosis (TB) from 2005 to 2014. Global and local Moran's I, autocorrelation analysis along with Ord $\left(\mathrm{Gi}^{*}\right)$ statistics was applied to detect spatial patterns and the hotspot of TB incidence. Furthermore, the Kuldorff's scan statistics were used to analyze space-time clusters. A total of 40,878 TB cases were reported in Beijing from 2005 to 2014 . The annual average incidence rate was 22.11 per 100,000 populations (ranged from 16.55 to 25.71). The seasonal incidence occurred from March to July until late autumn. A higher relative risk area for TB incidence was mainly detected in urban and some rural districts of Beijing. The significant most likely space-time clusters and secondary clusters of TB incidence were scattered diversely in Beijing districts in each study year. The risk population was mainly scattered in urban and dense populated districts, including in few rural districts.
\end{abstract}

\section{Keywords}

Tuberculosis, Spatial Statistics, Space-Time Analysis, Beijing, China 


\section{Introduction}

It has been established that Tuberculosis (TB) is a top killer chronic infectious disease in the world, where 10 million people were ill, and 1.6 million people died from the disease in 2017 [1]. Most of the deaths (95\%) occurred in low and middle-income countries, especially in South-East Asia and Western Pacific region [2]. China has the world's second largest TB epidemic country after India among the 22 TB high-burden countries [3]. In 1990, TB was the number one cause of death (360,000 people died) in the adult population in China [4], which has been reduced with the mortality dropping rate by 19.1 to $3.9 / 100,000$ individual populations since 1990 to 2010 [4]. TB is a top five ranked notifiable disease classified in China [5], that is why it is still a major public health issue in the country.

Geographic Information System (GIS) and its application in epidemiology for the disease interventions have been broadly utilized in spatial epidemiology [6]. It has been applied rapidly for disease investigation [7]. With an assistance of spatial statistics, spatial epidemiology attempts to describe and analyze the distribution of diseases, health conditions and latent factors too [8] [9]. Space-time scan statistic can describe the clustering characteristics in terms of both space and time [10] [11], which has been commonly used to detect and evaluate disease clusters [10] [12] for the variety of diseases [13] [14]. Furthermore, analyzing the clustering features of a disease can help to detect significant hotspots and high-risk clusters in space and time that may help to make such policy and program to prevent and treatment the TB infection.

Previously several studies have been conducted about TB in the study region [15]-[20]; however, most of those studies were focused on the drug resistance of anti-tuberculosis treatment (ATT), molecular characterization of TB bacteria, and epidemiological characteristics of TB infection. Moreover, a spatial-temporal and space-time analysis of TB incidence seems to be lacking, because such a model might be helpful in determining the high-risk areas of disease incidence on specific time periods. Therefore, a more location-specific public health intervention, control and prevention to TB infection at district/county level could be introduced [21]. Thus, we conducted the spatial and space-time scan statistics analysis in Beijing region to explore the distribution characteristics and detect spatial and spatial-temporal clusters (hotspots) of TB incidence.

\section{Methodology}

\subsection{Study Area and Design}

A retrospective study conducted in Beijing region based on the reported new (smear positive) pulmonary tuberculosis cases from 2005 to 2014. Beijing, the capital city of China, is located at the northern tip of the North China plain at $39^{\circ} 54^{\prime} 50^{\prime \prime} \mathrm{N}$ and $116^{\circ} 23^{\prime} 30^{\prime \prime} \mathrm{E}$ surrounded by the mountains. A total area of Beijing has $16,410.54 \mathrm{Km}^{2}$ and consists 16 administrative districts, and all districts are covered by this study (Figure 1) [22]. 


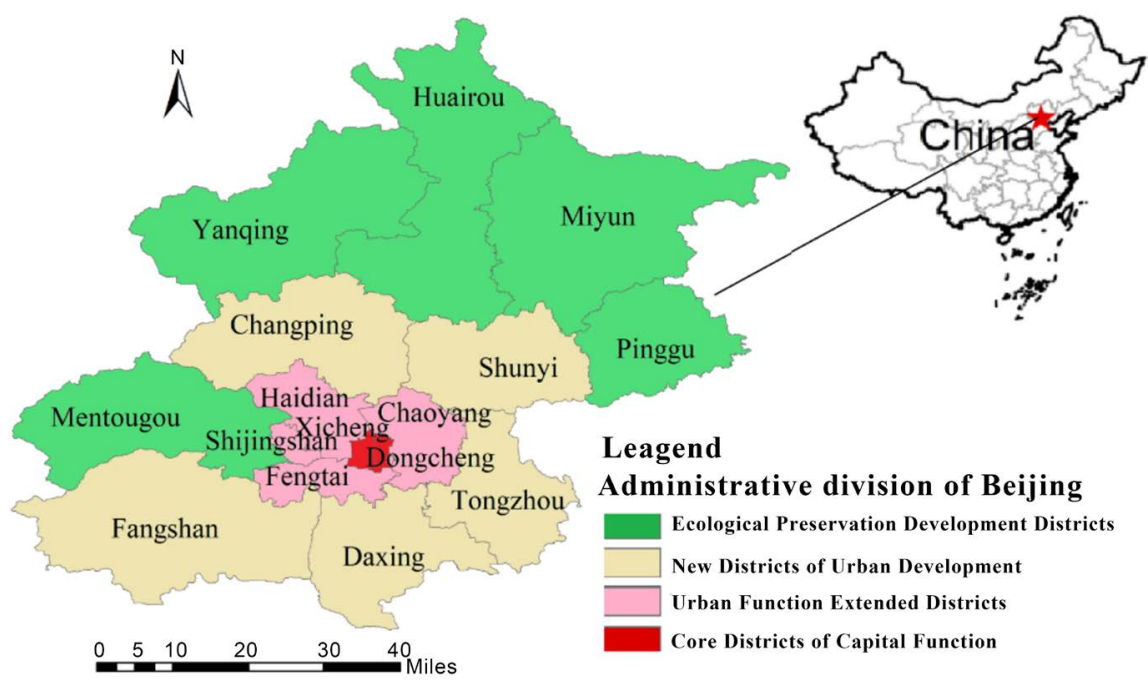

Figure 1. Map of the administrative districts based on their different urban functions and their locations in Beijing.

\subsection{Data Source}

All reported cases (smear positive cases) of TB data including age, sex, address, and occupation with clinical records (from 2005 to 2014) were obtained from the National Surveillance System for Notifiable Infectious Diseases, Beijing Information of Tuberculosis Prevention and Control registration database [23]. Demographic information (Population data) was collected from the Beijing $\mathrm{Mu}$ nicipal Bureau of Statistics, Beijing Statistical Yearbook, covering the year from 2005 to 2014 [22].

All TB cases were inputted into Microsoft Excel 2010 (Microsoft, Redwoods, WA, USA) and geo-coded according to their residential addresses and determining its longitude and latitude coordinate by using ArcGIS 10.1 (ESRI Inc, Redlands, CA USA) and linked to the respected location in Geographic Information system (GIS). To conduct a GIS-based analysis on the spatial distribution of TB, the districts/counties level polygon map at 1:100,000 scales were obtained from National Geometric Center of China [24].

\subsection{Ethical Review}

This study was approved under the Institutional Review Committee for Healthcare Research and Quality protocol at Capital Medical University, Beijing China, with secondary analysis of confidential data from the CDC, Beijing, China.

\subsection{Statistical Analysis}

\subsubsection{Descriptive Analysis}

We accumulated all TB cases by month with their diagnosis dates to identify the temporal patterns of the disease. We also summarized the TB cases including gender, age, occupation and the year of the diagnosis to identify the demographic characteristics of the disease by years. All descriptive information of 
respondents was summarized annually according to geographic area (districts) and all incidence rates were calculated for each neighborhood, using neighborhood census data as denominators and reported TB cases as the numerator. All descriptive analysis was conducted using SPSS software-v-20.

\subsubsection{Spatial Patterns Analysis}

Spatial autocorrelation (Global Moran's I) analysis was used to investigate the patterns of TB incidence. It evaluates whether its distribution pattern is clustered, dispersed or at random [25]. Moran's I index, Z scores and p-values were used to evaluate the significance of the test. In addition, Global Moran's I describe the trend of TB incidence patterns thus, we did each year analysis in the study region from 2005 to 2014. If the value of Global Moran's I Index near +1.0 indicates the clustering, which means the TB incidences should be similar among the neighboring districts, while near -1.0 indicates dispersion and zero mean total spatial randomness [26] [27]. Therefore, the spatial autocorrelation statistics (Global Moran's I) was used to distinguish the spatial autocorrelation of TB in the Beijing districts from 2005 to 2014 using ArcGIS 10.1 software

\subsubsection{Local Moran's I Statistics (LISA)}

We applied Anselin Local Moran's I (Local Indicators of Spatial Association-LISA) statistic to determine whether there are positive spatial correlations (high-high or low-low clusters) or negative spatial correlations (high-low or low-high clusters) exist. It also measures the spatial association between each individual spatial district with its neighboring districts [28]. In fact, this statistic was applied to detect the core clusters/outliers of districts with high TB incidences. Anselin spatial weights matrix is based on the inverse distance and weight between polygon centroids, which were constructed to detect the spatial relationships among the districts [28].

\subsubsection{Hot Spot Analysis}

To explore the additional information about the intensity and existence of core hotspots or cold spot cluster of TB incidence in the study location, we further assessed the local spatial autocorrelation analysis using Getis-Ord $\mathrm{Gi}^{*}$ statistic in ArcGIS software [29] [30]. This scan statistic assists as an indicator of the local autocorrelation, that measures how spatial autocorrelation varies locally over the study area and then calculates a statistic for each point data. This statistic provides more intuitive results with a better visual exploration and has an advantage of distinctive high value clusters (hot spot) or low value clusters (cold spot). The statistical significance of a Z-score dispensed to each district quantified the presence of hotspots and cold spots clusters of TB incidence, relative to the hypothesis of spatial uncertainty. The larger Z-score is the more penetrating the clustering of high values (hot spot) and vice-versa (cold spot) [29] [30]. In fact, if $\mathrm{Z}$ score $\left(\mathrm{Gi}^{\star}\right)$ is positive and significant, indicates that, this district and its neighboring districts have a relatively high occurrence of TB incidence, which is a hotspot, and contrary if the $\mathrm{Z}\left(\mathrm{Gi}^{*}\right)$ score is negative and significant, it indi- 
cates a cold-spot. Districts with a $\mathrm{Z}$ scores $>2.58$ were considered as significant at $99 \%$ confidence level $(\mathrm{p}<0.01$ ), and assumed in the hotspot category. Likewise, if Z-score between 1.65 - 1.96 and 1.96 - 2.58 were considered as significant at $90 \%$ confidence levels ( $\mathrm{p}<0.010$, and 0.05 ), and they were categorized in the high-risk districts. While, a Z-score with $<-2.58$ indicated that the clustering of low values, which means cold spot district. The Ord-Getis $\mathrm{Gi}^{\star}$ statistics has been described in the previous study [29] [30] [31].

\subsubsection{Space-Time Analysis}

A retrospective space-time scan analysis based on a discrete Poisson probability model was carried out using SaTScan ${ }^{\mathrm{TM}}$ V-9.4 software [32] to detect clusters of TB incidence in the study regions from 2005 to 2014. This method is defined by a cylindrical window with a circular geographic base and with the height corresponding to time [33]. This cylindrical window moves around the space and time scanning for clusters within the space-time window to compare the incidence rates of $\mathrm{TB}$ outside this window. The null hypothesis was that the relative risk (RR) of TB cases within the window was equal to that of outside. This study applied Poisson-based model to formulate the process of TB incidence regarding a known underlying population at risk.

In this study, the maximum size of space-time clusters was defined as twice higher risk at least within the window than outside $(R R \geq 2)$. First, people within the identified clusters are more likely to be infected with TB bacteria than those who identified within outside the clusters when the $R R$ is greater than one. So, this cluster is a risk factor for the TB infection. On the other hand, the cluster is a protective factor when the $R R$ is less than one. If there is no any effect for the risk on the infection, the $R R$ is one. Therefore, the selection for the maximum size of space-time cluster, needs the $R R>1$ at least. In this study, the spatial size of scanning window was setting as $50 \%$ of the total population at the risk. The statistical significance of each cluster in the study location was based on comparing the likelihood ratio (LLR) achieved from the Monte Carlo simulation with the maximum number of the replication set to 999 to ensure the sufficient statistical power. The window with the maximum LLR is assumed to be the most likely cluster that is the cluster least likely to be caused by chance, and other windows with a statistically significant LLR were measured as secondary clusters. The relative risk $(R R)$ of the incidence inside and outside the window considered to be statistically significant if $\mathrm{P}<0.05$ [32] [33].

In this study, the spatial units of space-time scan analysis were the 16 districts of Beijing, and the time units were 10 years from 2005 to 2014. The time frame of the scan analysis was set to be a month to control the time trends and to observe the cluster changes in the entire study period.

\section{Results}

\subsection{Basic Characteristics}

A total of 44,408 new sputum smear positive pulmonary tuberculosis cases were 
reported in Beijing from 2005 to 2014 . We excluded 3530 cases due to unavailable residence information or not residing in the study area. Finally, we employed 40,878 cases for the final analysis. The annual average incidence rate of TB observed as 22.11 per 100,000 populations, which is appeared almost stable from 2006 to 2008 , (25.65, to 25.71 per 100,000 populations), and displayed lowest in 2014 with 16.55/100,000 populations (Figure 2).

Likewise, the high incidence of TB was observed in highly densely populated districts (Figure 3), for example, Xicheng district. Moreover, TB incidence rates were noted high in Xicheng and Mentougou districts during the whole study period. The highest TB incidence found in the year 2005 in four districts, followed by 3 districts in 2007 to 2009, and only one district in 2014 .

\subsection{Temporal Patterns of TB Cases}

Figure 4 displays the seasonal patterns of TB occurrence in Beijing region from 2005 to 2014, where lowest seasonal incidence pattern can be observed in the month of February and October and remaining months have had almost similar pattern in each study year (Figure 4).

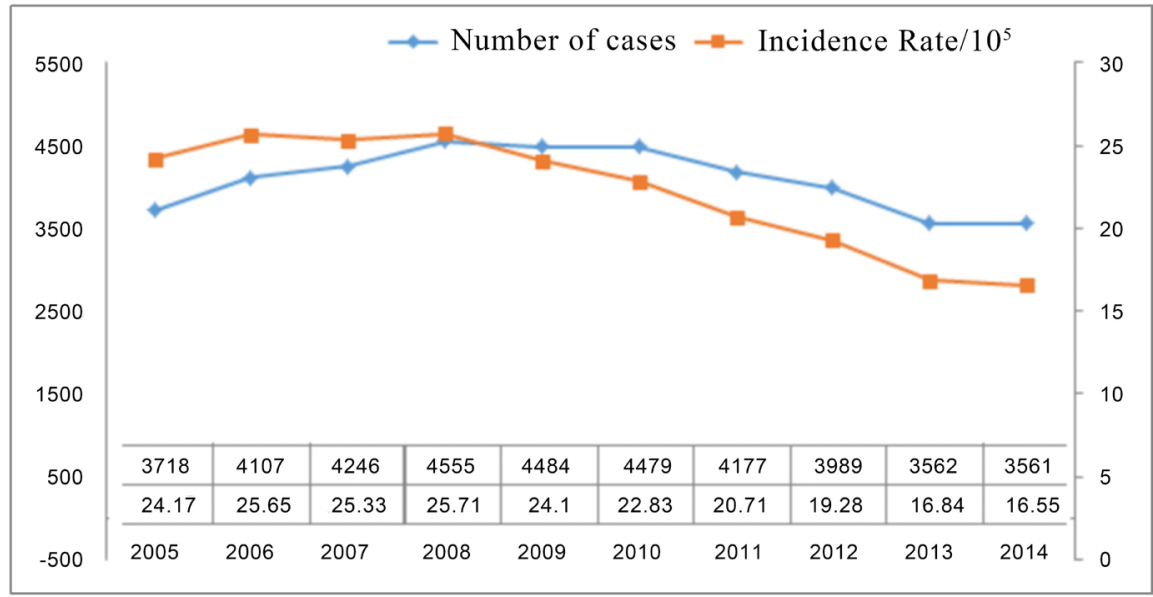

Figure 2. Tuberculosis incidence in Beijing districts from 2005-2014.

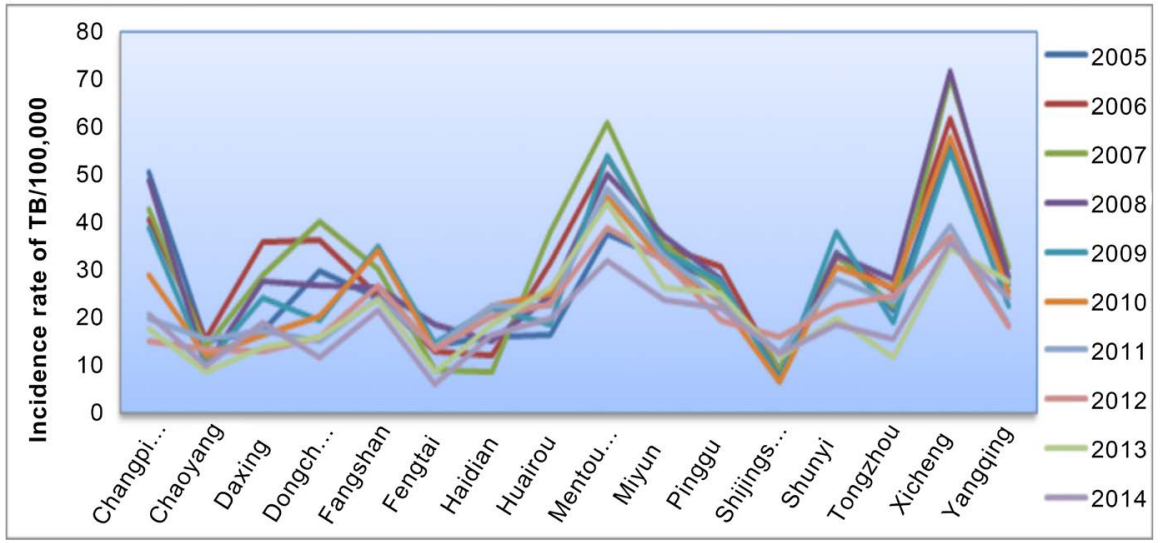

Figure 3. Distribution of TB incidence in Beijing districts, 2005-2014. 


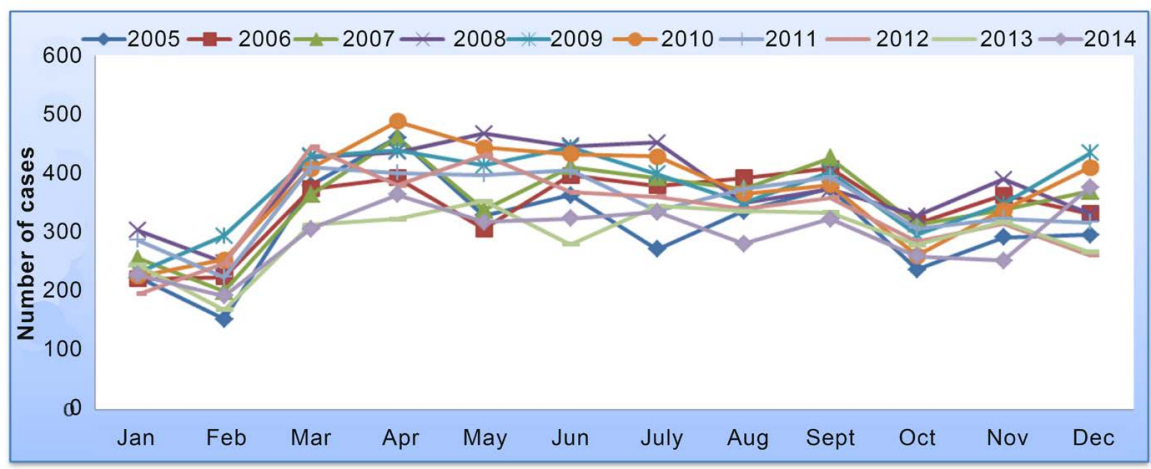

Figure 4. Temporal distribution of $\mathrm{TB}$ incidence across in the Beijing districts, 2005-2014.

\subsection{Spatial Pattern of TB Cases}

Global Moran's I autocorrelation analysis was used to detect TB incidence patterns such as clustered, dispersed and randomly distributed in the study region. The global spatial correlation analysis exhibited the presence of positive spatial clustering of TB incidence in Beijing region (Z-score from 0.842 to 2.324) (Table $1)$.

Anselin's Local Moran's I analysis disclosed the diverse patterns of the core clustering of high TB incidence districts with HH (high-high) and LH (low-high) cluster during the study period (Figure 5). Our findings suggest that the statistically significant geographic clustering of high tuberculosis incidence in center located districts in Beijing city (Figure 5, A-J).

The clusters of TB incidences, including hotspots and cold spots were estimates using the local Getis-Ord-Gï statistics. Location and size of hotspots clusters varied in each study year. The most hotspots incidence locations of TB incidence were detected in the urban districts e.g., Chaoyang, Xicheng, Fengtai and Dongcheng districts. The hotspots cluster further divided into primary (GiZ score $>2.58)$, secondary (GiZ score $=1.96-2.58 \mathrm{SDs}$ ) and tertiary (GiZ score $=$ 1.64 - 1.96) according to the values of GiZ score for the clearly defining the intensity of clusters (Figure 6). The average primary hotspot clusters, including secondary hot spot clusters districts (Haidian, Shijinshan, Tongzhou and Changping) are scattered in the Figure 6.

\subsection{Space-Time Analysis of TB in Beijing}

A Space-time cluster analysis of TB incidence was conducted in Beijing districts by using space-time permutation model to identify the statistically significant monthly spatiotemporal clusters from 2005-2014 (Table 2).

The most likely clusters of TB incidence were detected varied in each study year. A large circular most likely cluster was detected in the northern part of Beijing covering three districts (Yanqing, Huairou, and Miyun) with cluster time ranged from $1^{\text {st }}$ to $31^{\text {st }}$ January, clusters center were $40.628^{\circ} \mathrm{N}, 116.578^{\circ} \mathrm{E}$, and $40.524^{\circ} \mathrm{N}, 116.986^{\circ} \mathrm{E}$, radius $37.05 \mathrm{Km}$ and $37.73 \mathrm{Km}$ with the relative risk $(R R)$ 
Table 1. Global spatial autocorrelation analysis of TB incidences in Beijing, 2005-2014.

\begin{tabular}{cccc}
\hline Years & Moran's-I & Z Score & P-Value \\
\hline 2005 & 0.199 & 1.920 & 0.033 \\
2006 & 0.235 & 2.324 & 0.017 \\
2007 & 0.118 & 1.627 & 0.059 \\
2008 & 0.064 & 1.072 & 0.149 \\
2009 & 0.054 & 0.842 & 0.191 \\
2010 & 0.115 & 1.263 & 0.103 \\
2011 & 0.149 & 1.623 & 0.066 \\
2012 & 0.163 & 1.751 & 0.051 \\
2013 & 0.100 & 1.354 & 0.107 \\
2014 & 0.104 & 1.142 & 0.119 \\
\hline
\end{tabular}
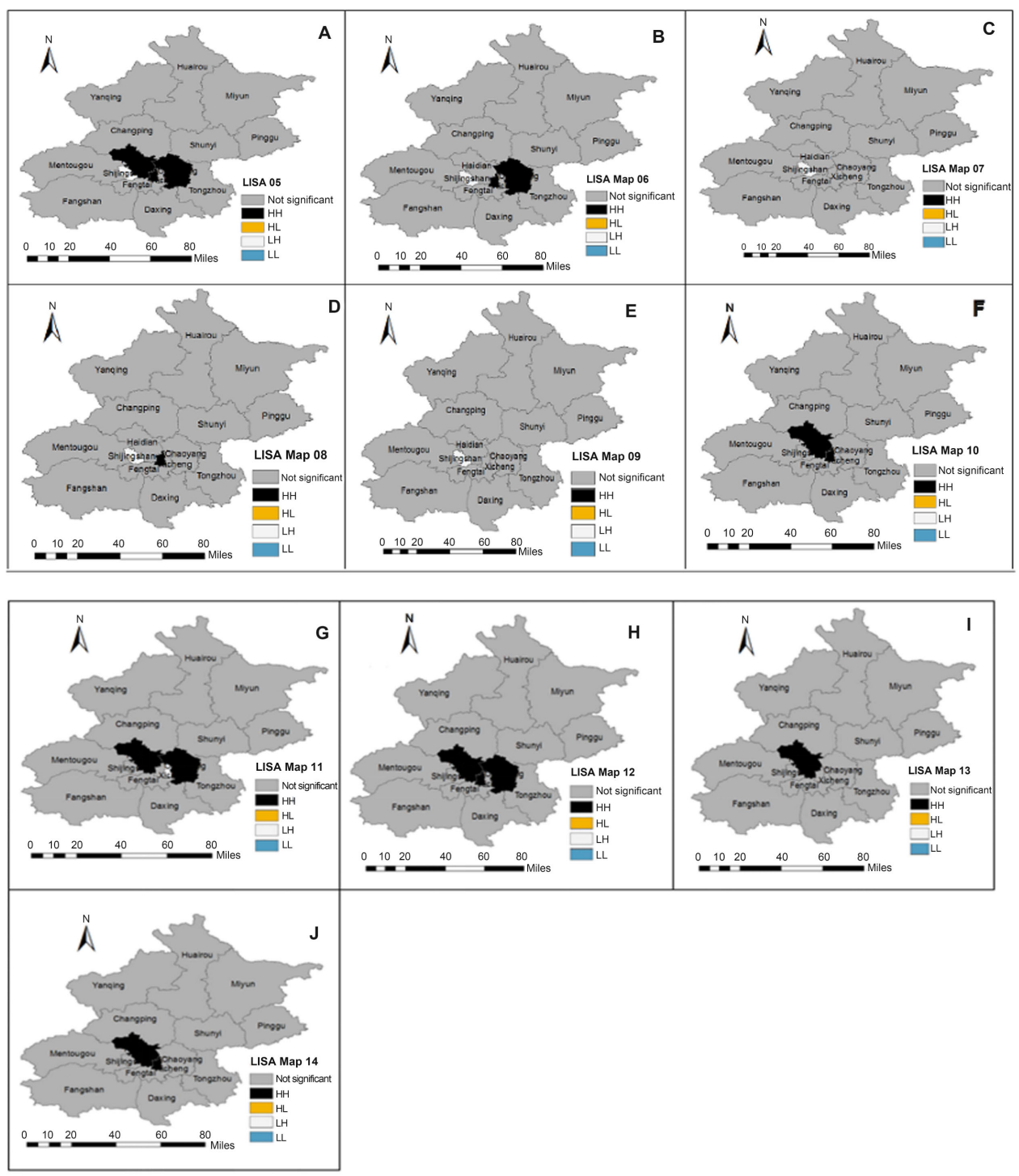

Figure 5. Local Moran's I cluster map of TB incidence in Beijing districts (A-J respectively from 2005-2014). 

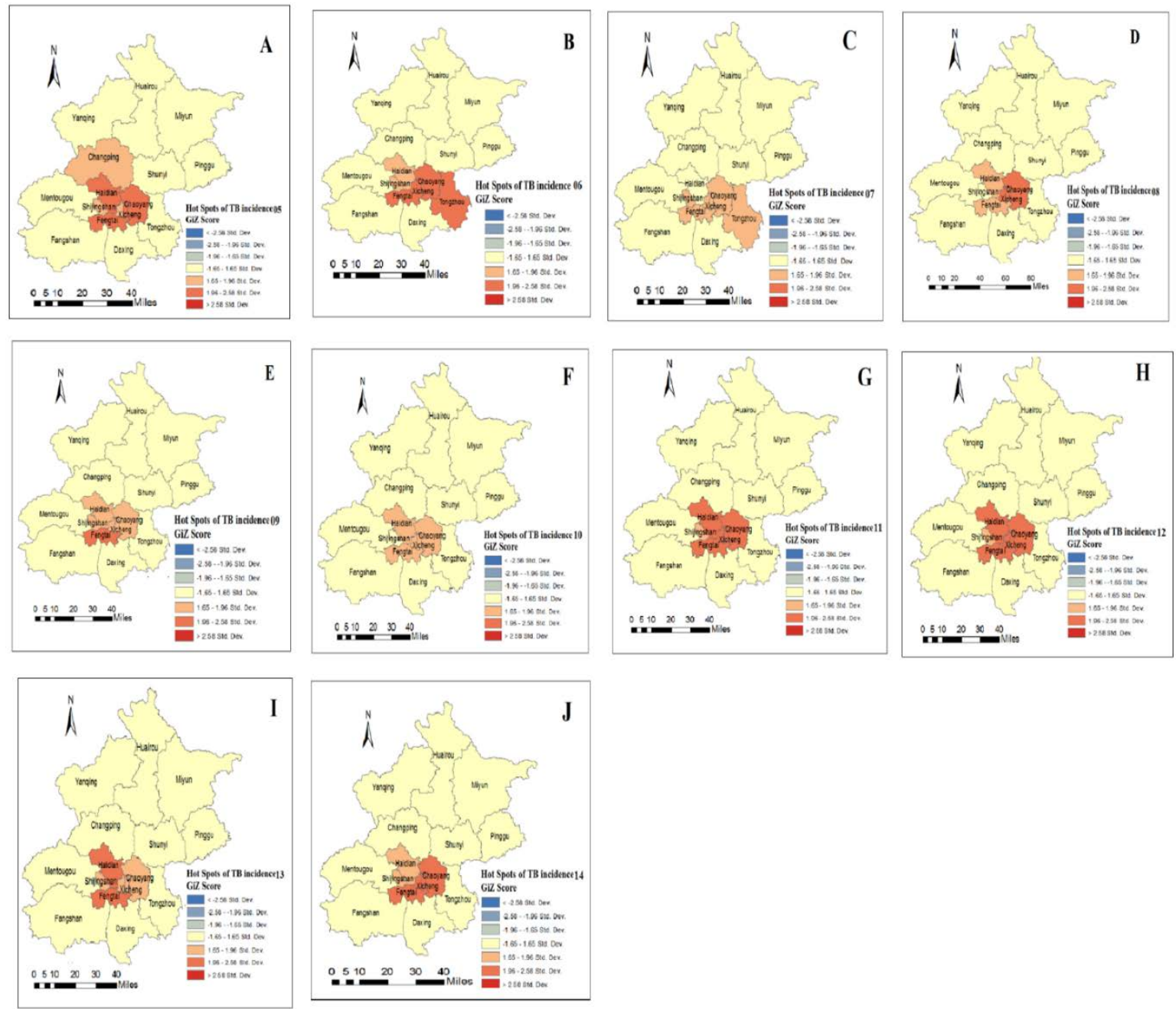

Figure 6. Hotspot clusters of TB incidence in Beijing, 2005-2014.

Table 2. The most likely high-risk clusters of tuberculosis incidence detected using a space-time permutation model (setting $50 \%$ as the maximum cluster size) in Beijing, 2005-2014

\begin{tabular}{cccccc}
\hline $\begin{array}{c}\text { Scan } \\
\text { Year }\end{array}$ & $\begin{array}{c}\text { Cluster Time } \\
\text { Frame }\end{array}$ & $\begin{array}{c}\text { Center (Latitude, } \\
\text { Longitude)/Radius }(\mathrm{km})\end{array}$ & $\begin{array}{c}\text { Cluster } \\
\text { Districts }\end{array}$ & $\begin{array}{c}\text { Relative } \\
\text { Risk }\end{array}$ & P-Value \\
\hline 2005 & $01-31 \mathrm{Jan}$ & $116.5787 \mathrm{E}, 40.628 \mathrm{~N} / 36.25 \mathrm{~km}$ & 2 & 0.23 & $<0.001$ \\
2006 & $01-31 \mathrm{Jan}$ & $40.6281 \mathrm{~N}, 116.578 \mathrm{E}) / 36.25 \mathrm{~km}$ & 2 & 2.29 & $<0.001$ \\
2007 & $01-31 \mathrm{Jan}$ & $40.524 \mathrm{~N}, 116.986 \mathrm{E} / 0 \mathrm{~km}$ & 1 & 0.16 & $<0.001$ \\
2008 & 01 Nov - 31 Dec & $39.911 \mathrm{~N}, 116.410 \mathrm{E} / 0 \mathrm{~km}$ & 1 & 2.03 & $<0.001$ \\
2009 & $01-31 \mathrm{Jan}$ & $40.6281 \mathrm{~N}, 116.578 \mathrm{E} / 37.05 \mathrm{~km}$ & 3 & 0.30 & $<0.001$ \\
2010 & $01-31 \mathrm{Jan}$ & $40.524 \mathrm{~N}, 116.986 \mathrm{E} / 37.73 \mathrm{~km}$ & 3 & 0.20 & $<0.001$ \\
2011 & $01-31 \mathrm{Jan}$ & $40.214 \mathrm{~N}, 116.204 \mathrm{E} / 21.03 \mathrm{~km}$ & 2 & 2.89 & $<0.001$ \\
2012 & $01-31 \mathrm{Jan}$ & $40.214 \mathrm{~N}, 116.204 \mathrm{E} / 21.03 \mathrm{~km}$ & 2 & 2.84 & $<0.001$ \\
2013 & $01-31 \mathrm{Jan}$ & $40.214 \mathrm{~N}, 116.204 \mathrm{E} / 21.03 \mathrm{~km}$ & 2 & 0.29 & $<0.001$ \\
2014 & $01-31 \mathrm{Jan}$ & $40.214 \mathrm{~N}, 116.204 \mathrm{E} / 21.03 \mathrm{~km}$ & 2 & 3.47 & $<0.001$ \\
\hline
\end{tabular}


value 0.30 and $0.20(\mathrm{P}<0.001)$ respectively in 2009 and 2010 . Followed by second largest circular clusters were detected in 2005 and 2006 covering two districts (Huairou and Miyun district), and similarly, Haidian and Changping district in 2013 and 2014, whereas only one small cluster was found in Miyun and Xicheng district in 2007 and 2008 respectively in the study region. There were several secondary clusters were also detected apart from the most likely clusters (Figure 7).

This study declared that the most likely and secondary clusters of TB incidence were scattered both in rural and urban districts in Beijing (Figure 7), indicating that people who resides high risk (most likely cluster) area are more

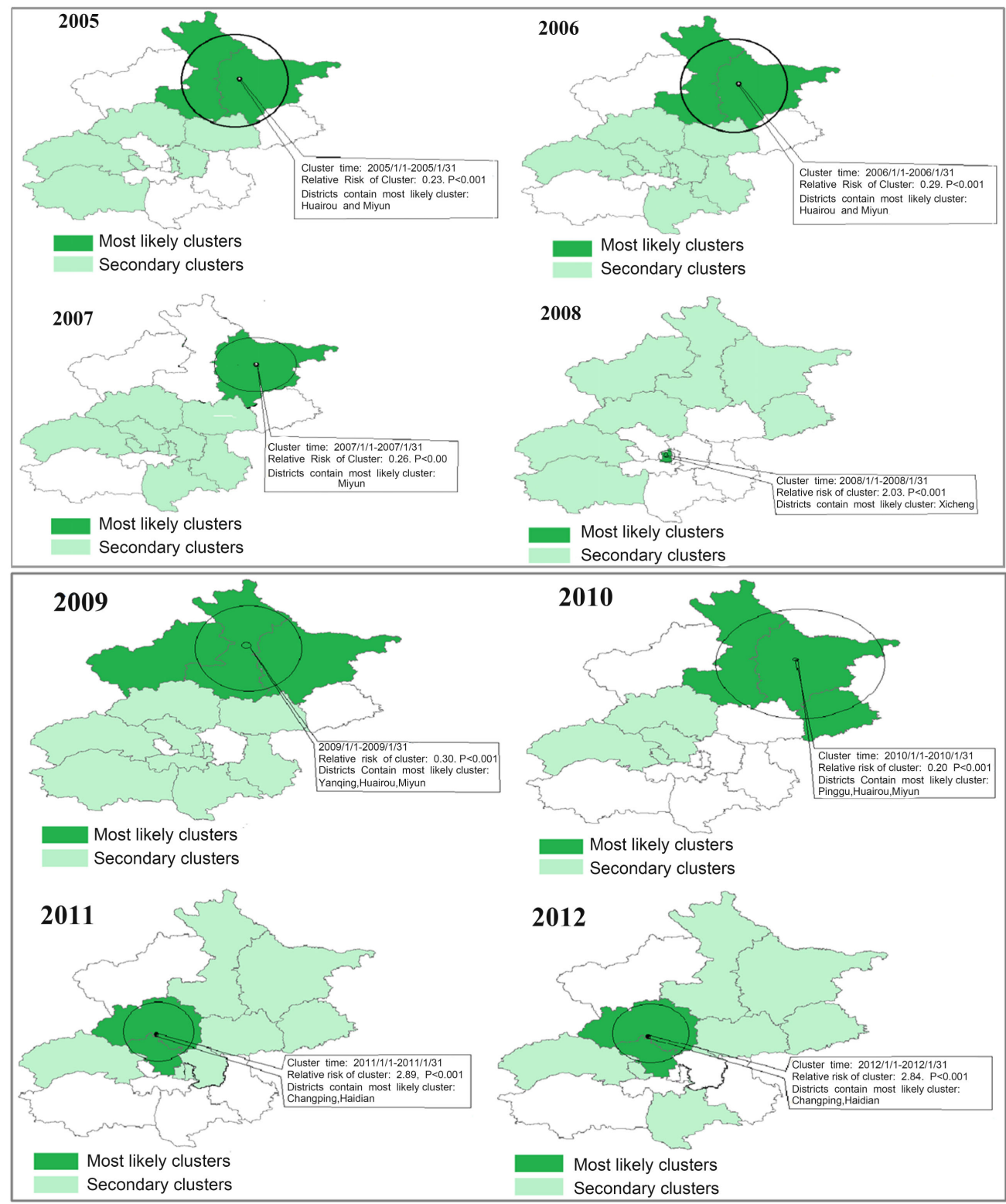

Figure 7. The most likely clusters and secondary clusters of TB incidence in Beijing were detected using a space-time permutation model, 2005-2014. Green indicates the most likely clusters, slightly green indicates secondary clusters, and white indicates no clusters in the study area. 
likely to prone to have a disease infection than those who reside outside the low relative risk area. The study found the most likely cluster of TB incidence with high Relative Risk values $(R R>1)$ in the year 2006, 2008, 2011-2012 and 2014, whereas low relative risk cluster of TB incidence with low RR values $(<1)$ were detected in other remaining years (Figure 7 and Table 2).

\section{Discussion}

We investigated the spatial patterns and space-time clusters of TB incidence and highlighted those geographic areas in Beijing districts from 2005 to 2014. This study established that Xicheng district followed by Mentouhou district had the highest TB incidence during in the study period (Figure 3). This study also identified the hotspots of TB incidence in the urban districts such as Chaoyang, Haidian, Xicheng, Fengtai, and Dongcheng (Figure 5, Figure 6). Basically, these districts are densely populated districts compare to rest districts in Beijing, which is consistent with the finding of the previous studies [34] [35] [36]. As per the national level, TB incidence rate was declined by 2.2\% between 1990 to 2000 , and $4.7 \%$ between 2000 to 2010 reported by the Representative office of WHO China [37].

The declining of TB incidence was noted since 2006 in the study region, that could be due to apply the standard diagnosis and treatment system with improved notification of TB cases after setup the MDGs Goal in the year 2000. This declining of TB incidence reveals the effectiveness of TB control program and implementation of Direct Observed Treatment Short-Course (DOTS) strategy in district level over the last two decades [2]. Recently, Beijing government has expanded the range of free treatment service to TB patients, including alleviated the inspection charges too for those who are not permanent residents in Beijing region, whereas, public health and welfare service was restricted previously to those who were not permanent residents in Beijing region [36]. Likewise, Beijing Research Institute of Tuberculosis Control has established a TB prevention and control institute in each district in Beijing to provide the convenient TB treatment to all [38].

Through this study detected seasonal trends with apparent peaks in all season. Almost, the disease incidence peaks were detected in summer between March to July until late autumn and in winter. Such seasonal patterns revealed by the previous studies in different regions as like: in China [39] [40], Hong Kong [40] [41], Taiwan [42], India [43], and United Kingdom [44]. In winter session, especially from January to February and in October found low incidence peak, that may be due to the holiday for the Chinese New Year festival and National Day of China respectively, where each school/college, government or non-governmental organizations are closed, and go back to home for the celebration of festival with family and friend. In this regard, the population density of Beijing may be decreased and incidence of TB became low. To know the extensive reason for this seasonal pattern, there is needed a further ecological study including meteoro- 
logical parameters.

The estimates of the global Moran's I indicated that occurrence of TB was distributed randomly at the district level, which is consistent with the finding of Lan Li et al. [34]. Likewise, statistically significant TB incidence clusters, including hot spots clusters were detected in the different districts in each study year. We identified high-high TB incidence clusters districts, including hotspots clusters in the urban functional districts such as; Chaoyang, Haidian, Xicheng, Dongcheng and Fengtai, where population density is high compared to rest districts in Beijing. This finding is steady with the study of Y. Liu, et al. [35], which established that the TB occurrence was high in urban development new districts and ecological reservation development districts during 2005 to 2009. Contrast finding revealed by the study of Lan $\mathrm{Li}$, et al., [34] that TB occurrence was detected high in Chaoyang and Daxing districts and overall occurrence of TB across the districts in Beijing was relatively low and steady over the study year, 2009 to 2014.

The analysis of space-time scan statistic highlighted the significant most likely and several secondary clusters, which were distributed diversely in each study year. Three most likely clusters were detected in Ecological Preservation Development districts in 2009 (Yanqing, Huairou, Miyun) and 2010 (Huairou, Miyun, and Pinggu districts) and followed by two most likely clusters in 2005 and 2006 such as g, Huairou, and Miyun, districts (Figure 7). These clusters were scattered in the relatively poor rural districts where TB diagnosis and treatment facilities were less available compared to other urban districts. These distribution characteristics of the disease incidence clusters were consistent with the previous studies which were conducted in different countries [8] [45] [46] [47] [48], urged the existence of both spatial and space-time clusters in the same locations, which supports the evidence of uneven distribution of TB disease. In contrast, the study of Lan Li, et al. [34] detected the most likely cluster in Core capital function district e.g. Xicheng from 2009 to 2011 in the same study area. However, secondary significant clusters were detected in same districts as our study and Lan, Li et al.'s study [34]. In addition, two more large most likely clusters with high RR values were identified in New districts of Urban Development and Urban Function Extended districts such as; Changing and Haidian from 2011 to 2014. This finding is similar as the study of Y Liu, et al., [35] and Lan, Li, et al., [34]. A study conducted in Ethiopia also supported that most likely clusters were identified in the urban and rural area where population density, as well as the area close to towns and near to road network (which linked to major towns) [46].

Several studies have stated that overcrowding conditions and social disturbance could all be risk factors for the burden and variations of disease occurrence. Furthermore, patient care factors [49], as well as poor health care and TB control services may also contribute to the high rate of the disease occurrence [9] since infectious cases may persist undiagnosed and may not obtain treat- 
ment, which could subsequently contribute to the transmission dynamics of the disease. Additionally, most urban districts where the clusters identified are the centers of the districts, market hubs, business areas, public transportation routes and hub for the different socio-economic activities. To better access the road and movement using public transportation in a crowded and poorly ventilated area may assist in facilitating contact with TB infection, that could favor a spread of the disease in the locations where clusters were identified [50].

Spatial analysis has been extensively utilized to detect the distribution patterns of various communicable diseases along with non-communicable diseases by GIS technology, ArcGIS, SaTScan and other relevant software, and assimilated meaningful results [51]. Investigators can identify the patterns and relationships in the data based on geography through spatial analysis [52]. Significant hotspot clusters including most likely clusters and secondary clusters were displayed in this study prioritized for public health action according to the statistical analysis [53]. The methods applied in this study contribute to TB control and prevention in Beijing region, and then the resources allocated for TB control can be repositioned according to the analysis. The detection of TB risk areas using the surveillance data based on the geographic community is a relatively inexpensive task [54]. An improved surveillance and an improved access to utilization of TB control services may help to increase TB case identification that could contribute to reducing the disease transmission, which might also affect the disease notification rates. Further intervention is therefore needed to better understand the role of health service access to the case identifications and clustering of pulmonary $\mathrm{TB}$ in the study region.

Though our study demonstrated the usefulness of spatial and temporal clustering analysis in Beijing, where there were some limitations. First, the estimated risk of TB infection might be underestimated in some areas because cases may not be reported or recorded in the health system. Second, we used space-time scan statistics to detect clusters in different space and time. The method that depends on circular spatial scanning windows and space-time cylinders, that does not allow for irregular space [55]. Lastly, potential risk factors that could be related to the clustering were not evaluated in this study. We only analyzed the spatial-temporal patterns of TB incidence and clusters. However, the high prevalence of TB may be related to individual and socio-economic factors, poor living condition, low economic status, exposure to ambient air pollution, and some meteorological factors. Therefore, further studies can be accompanied to cover the roles of these factors in the spread of TB disease.

\section{Conclusion}

We investigated the space-time scan characteristic of pulmonary TB incidence in Beijing region and identified the hotspot clusters in urban and some rural districts over the study period by using GIS analysis. The most likely significant clusters and relative risk of TB were detected by space-time analysis in the urban 
districts and few clusters in rural districts. Therefore, our findings suggest that TB control measures should be focused on those "hot spot" districts including most likely clusters districts to allocate public health resources more precisely to reduce the burden of TB incidence.

\section{Acknowledgements}

The authors thank the Ministry of Science and Technology of Beijing, China, National Center for Public Health Surveillance and Information Services, China Center for the Disease Control and Prevention, Beijing, China for providing the tuberculosis case data.

\section{Author Contributions}

Conceptualization, Gehendra Mahara and Xiuhua Guo; Data curation, Gehendra Mahara, Mina Karki, Kun Yang and Sipeng Chen; Formal analysis, Gehendra Mahara, Kun Yang and Sipeng Chen; Methodology, Gehendra Mahara and Mina Karki; Project administration, Xiuhua Guo; Resources, Kun Yang and Xiuhua Guo; Software, Gehendra Mahara and Kun Yang; Writing-original draft, Gehendra Mahara; Wei Wang, Writing_review \& editing, Gehendra Mahara, Xiuhua Guo and Wei Wang.

\section{Conflicts of Interest}

We the authors declare that we have no conflict of interests.

\section{References}

[1] World Health Organization (WHO) (2018) Factsheet of Tuberculosis (TB). http://www.who.int/news-room/fact-sheets/detail/tuberculosis

[2] Tian, H. and Wenjie, S. (2013) Tuberculosis in China. Journal of Tuberculosis Research, 1, 9-10. https://doi.org/10.4236/jtr.2013.12002

[3] Liu, J.J., Yao, H.Y. and Liu, E.Y. (2005) Analysis of Factors Affecting the Epidemiology of Tuberculosis in China. International Journal of Tuberculosis and Lung Disease, 9, 450-454.

[4] Disease Control Bureau of the Ministry of Health (2010) Report on the 5th National Tuberculosis Epidemiological Survey in China. 30-37.

[5] Sun, W., Gong, J., Zhou, J., Zhao, Y., Tan, J., Ibrahim, A.N. and Zhou, Y. (2015) A Spatial, Social And Environmental Study of Tuberculosis in China Using Statistical and GIS Technology. International Journal of Environmental Research and Public Health, 12, 1425-1448. https://doi.org/10.3390/ijerph120201425

[6] Zhou, X. (2009) Spital Epidemiology. Science Press, Beijing.

[7] Pfeiffer, D., Robinson, T. and Clements, A. (2008) Spatial Clustering of Disease and Global Estimates of Spatial Clustering. Oxford University Press, New York. https://doi.org/10.1093/acprof:oso/9780198509882.003.0004

[8] Onozuka, D. and Hagihara, A. (2007) Geographic Prediction of Tuberculosis Clusters in Fukuoka, Japan, Using the Space-Time Scan Statistic. BMC Infectious Diseases, 7, 26-26. https://doi.org/10.1186/1471-2334-7-26

[9] Spatial Dependency of Tuberculosis Incidence in Taiwan (2018). 
https://journals.plos.org/plosone/article?id=10.1371/journal.pone.0050740

[10] Kulldorff, M. and Nagarwalla, N. (1995) Spatial Disease Clusters: Detection and Inference. Statistics in Medicine, 14, 799-810. https://doi.org/10.1002/sim.4780140809

[11] Kulldorff, M. (1997) A Spatial Scan Statistic: Communications in Statistics. Theory and Methods, 26, 1481-1496. https://doi.org/10.1080/03610929708831995

[12] Kulldorff, M. (2001) Prospective Time Periodic Geographical Disease Surveillance Using a Scan Statistic. Journal of the Royal Statistical Society: Series A (Statistics in Society), 164, 61-72. https://doi.org/10.1111/1467-985X.00186

[13] Michelozzi, P., et al. (2002) Adult and Childhood Leukemia near a High-Power Radio Station in Rome, Italy. American Journal of Epidemiology, 155, 1096-103. https://doi.org/10.1093/aje/155.12.1096

[14] Green, C., et al. (2003) Geographic Analysis of Diabetes Prevalence in an Urban Area. Social Science \& Medicine, 57, 551-560. https://doi.org/10.1016/S0277-9536(02)00380-5

[15] Gao, G., Lian, L., Sun, Y., Wei, J., Xiao, J., Wang, X., Zhang, L., Zhao, X., Yang, D., Zhao, H., et al. (2015) Drug Resistance Characteristics of Mycobacterium Tuberculosis Isolates to Four First-Line Antituberculous Drugs from Tuberculosis Patients with AIDS in Beijing, China. International Journal of Antimicrobial Agents, 45, 124-129. https://doi.org/10.1016/j.ijantimicag.2014.09.013

[16] Li, F., Li, H., Zuo, W.-Z., Mi, L., Wang, X., Wang, Y., Wang, H., Shen, A., Cao, S. and Yuan, L. (2015) The Viability and Protein Expression of Beijing/W Lineage Mycobacterium tuberculosis Circulating in Xinjiang, China. Current Microbiology, 70, 735-744. https://doi.org/10.1007/s00284-015-0776-Z

[17] Li, G.-L., Zhao, D.-F., Xie, T., Ju, H.-F., Mu, C., Zhao, H. and Wang, X.-X. (2010) Molecular Characterization of Drug-Resistant Beijing Family Isolates of Mycobacterium tuberculosis from Tianjin, China. Biomedical and Environmental Sciences, 23, 188-193. https://doi.org/10.1016/S0895-3988(10)60051-7

[18] Li, X., Xu, P., Shen, X., Qi, L., DeRiemer, K., Mei, J. and Gao, Q. (2011) Non-Beijing Strains of Mycobacterium tuberculosis in China. Journal of Clinical Microbiology, 49, 392-395. https://doi.org/10.1128/JCM.00754-10

[19] Ying, L.X., Ying, L., Zhang, Y., Kang, W.L., Zhao, L. and Wei, M. (2015) The Epidemiological Characteristics of Beijing Lineage Mycobacterium tuberculosis from a National Referral Center in China. Biomedical and Environmental Sciences, 28, 538-343.

[20] Lu, B., Zhao, P., Liu, B., Dong, H., Yu, Q., Zhao, X. and Wan, K. (2012) Genetic Diversity of Mycobacterium tuberculosis Isolates from Beijing, China Assessed by Spoligotyping, LSPs and VNTR Profiles. BMC Infectious Diseases, 12, 372. https://doi.org/10.1186/1471-2334-12-372

[21] Lawson, A.B. (2006) Statistical Methods in Spatial Epidemiology. 2nd Edition, John Wiley \& Sons, Inc., Hoboken.

https://www.wiley.com/en-us/Statistical+Methods+in+Spatial+Epidemiology\%2C+ 2nd+Edition-p-9780470014844 https://doi.org/10.1002/9780470035771

[22] (2015) Beijing Statistical Yearbook. Beijing Municipal Bureau of Statistics, Beijing.

[23] (2017) National Surveillance System for Notifiable Diseases. Beijing Information of Tuberculosis Prevention and Control Registration Database, Central for Disease Control and Prevention (CDC), Beijing.

[24] National Earth System Science Data Sharing Platform. 
http://www.geodata.cn/

[25] Getis, A. (1991) Spatial Interaction and Spatial Autocorrelation: A Cross-Product Approach. Environment and Planning A: Economy and Space, 23, 1269-1277.

https://journals.sagepub.com/doi/abs/10.1068/a231269\#articleCitationDownloadCo ntainer https://doi.org/10.1068/a231269

[26] Mahara, G., Wang, C., Yang, K., Chen, S., Guo, J., Gao, Q., Wang, W., Wang, Q. and Guo, X. (2016) The Association between Environmental Factors and Scarlet Fever Incidence in Beijing Region: Using GIS and Spatial Regression Models. International Journal of Environmental Research and Public Health, 13, 1083. https://doi.org/10.3390/ijerph13111083

[27] Cliff, A.D. and Ord, K. (1970) Spatial Autocorrelation: A Review of Existing and New Measures with Applications. Economic Geography, 46, 269-292.

https://doi.org/10.2307/143144

[28] Anselin, L. (1995) Local Indicators of Spatial Association-LISA. Geographical Analysis, 27, 93-115.

https://onlinelibrary.wiley.com/doi/epdf/10.1111/j.1538-4632.1995.tb00338.x https://doi.org/10.1111/j.1538-4632.1995.tb00338.x

[29] Ord, J.K. and Getis, A. (1995) Local Spatial Autocorrelation Statistics: Distributional Issues and an Application. Geographical Analysis, 27, 286-306. https://onlinelibrary.wiley.com/doi/epdf/10.1111/j.1538-4632.1995.tb00912.x https://doi.org/10.1111/j.1538-4632.1995.tb00912.x

[30] JK, O. and Arthur, G. (2001) Testing for Local Spatial Autocorrelation in the Presence of Global Autocorrelation. Journal of Regional Science, 41, 411-432. https://doi.org/10.1111/0022-4146.00224

[31] Mahara, G., Wang, C., Huo, D., Xu, Q., Huang, F., Tao, L., Guo, J., Cao, K., Long, L., Chhetri, J.K., et al. (2016) Spatiotemporal Pattern Analysis of Scarlet Fever Incidence in Beijing, China, 2005-2014. International Journal of Environmental Research and Public Health, 13, 131. https://doi.org/10.3390/ijerph13010131

[32] SaTScan. Software for the Spatial, Temporal, and Space-Time Scan Statistics. https://www.satscan.org/

[33] Martin, K. (2018) SaTScan ${ }^{\mathrm{TM}}$ User Guide for Version 9.6.

[34] Li, L., Xi, Y. and Ren, F. (2016) Spatio-Temporal Distribution Characteristics and Trajectory Similarity Analysis of Tuberculosis in Beijing, China. International Journal of Environmental Research and Public Health, 13, 291. https://doi.org/10.3390/ijerph13030291

[35] Liu, Y., Li, X., Wang, W., Li, Z., Hou, M., He, Y., Wu, W., Wang, H., Liang, H. and Guo, X. (2012) Investigation of Space-Time Clusters and Geospatial Hot Spots for the Occurrence of Tuberculosis in Beijing. The International Journal of Tuberculosis and Lung Disease, 16, 486-491. https://doi.org/10.5588/ijtld.11.0255

[36] Jia, Z.-W., Jia, X.-W., Liu, Y.-X., Dye, C., Chen, F., Chen, C.-S., Zhang, W.-Y., Li, X.-W., Cao, W.-C. and Liu, H.-L. (2008) Spatial Analysis of Tuberculosis Cases in Migrants and Permanent Residents, Beijing, 2000-2006. Emerging Infectious Diseases, 14, 1413-1419. https://doi.org/10.3201/eid1409.071543

[37] World Health Organization, Western Pacific Regional Office (WPRO) Tuberculosis in China. http://www.wpro.who.int/china/mediacentre/factsheets/tuberculosis/en/

[38] Beijing Tuberculosis Control Research Institute. http://www.bjjks.org/

[39] Ge, E., Zhang, X., Wang, X. and Wei, X. (2016) Spatial and Temporal Analysis of 
Tuberculosis in Zhejiang Province, China, 2009-2012. Infectious Diseases of Poverty, 5, 11. https://doi.org/10.1186/s40249-016-0104-2

[40] Li, X.-X., Wang, L.-X., Zhang, H., Du, X., Jiang, S.-W., Shen, T., Zhang, Y.-P. and Zeng, G. (2013) Seasonal Variations in Notification of Active Tuberculosis Cases in China, 2005-2012. PLoS ONE, 8, e68102. https://doi.org/10.1371/journal.pone.0068102

[41] Leung, C.C., Yew, W.W., Chan, T.Y.K., Tam, C.M., Chan, C.Y., Chan, C.K., Tang, N., Chang, K.C. and Law, W.S. (2005) Seasonal Pattern of Tuberculosis in Hong Kong. International Journal of Epidemiology, 34, 924-930. https://doi.org/10.1093/ije/dyi080

[42] Liao, C.-M., Hsieh, N.-H., Huang, T.-L., Cheng, Y.-H., Lin, Y.-J., Chio, C.-P., Chen, S.-C. and Ling, M.-P. (2012) Assessing Trends and Predictors of Tuberculosis in Taiwan. BMC Public Health, 12, 29. https://doi.org/10.1186/1471-2458-12-29

[43] Thorpe, L.E., Frieden, T.R., Laserson, K.F., Wells, C. and Khatri, G.R. (2004) Seasonality of Tuberculosis in India: Is It Real and What Does It Tell Us? The Lancet, 364, 1613-1614. https://doi.org/10.1016/S0140-6736(04)17316-9

[44] Douglas, A.S., Strachan, D.P. and Maxwell, J.D. (1996) Seasonality of Tuberculosis: The Reverse of Other Respiratory Diseases in the UK. Thorax, 51, 944-946. https://doi.org/10.1136/thx.51.9.944

[45] Wang, T., Xue, F., Chen, Y., Ma, Y. and Liu, Y. (2012) The Spatial Epidemiology of Tuberculosis in Linyi City, China, 2005-2010. BMC Public Health, 12, 885. https://doi.org/10.1186/1471-2458-12-885

[46] Dangisso, M.H., Datiko, D.G. and Lindtjørn, B. (2015) Spatio-Temporal Analysis of Smear-Positive Tuberculosis in the Sidama Zone, Southern Ethiopia. PLoS ONE, 10, e0126369. https://doi.org/10.1371/journal.pone.0126369

[47] Gómez-Barroso, D., Rodriguez-Valín, E., Ramis, R. and Cano, R. (2013) Spatio-Temporal Analysis of Tuberculosis in Spain, 2008-2010. International Journal of Tuberculosis and Lung Disease, 17, 745-751. https://doi.org/10.5588/ijtld.12.0702

[48] Tiwari, N., Adhikari, C.M.S., Tewari, A. and Kandpal, V. (2006) Investigation of Geo-Spatial Hotspots for the Occurrence of Tuberculosis in Almora District, India, Using GIS and Spatial Scan Statistic. International Journal of Health Geographics, 5 , 33. https://doi.org/10.1186/1476-072X-5-33

[49] Randremanana, R.V., Richard, V., Rakotomanana, F., Sabatier, P. and Bicout, D.J. (2010) Bayesian Mapping of Pulmonary Tuberculosis in Antananarivo, Madagascar. BMC Infectious Diseases, 10, 21. https://doi.org/10.1186/1471-2334-10-21

[50] Mahara, G., Yang, K., Chen, S., Wang, W. and Guo, X. (2018) Socio-Economic Predictors and Distribution of Tuberculosis Incidence in Beijing, China: A Study Using a Combination of Spatial Statistics and GIS Technology. Medical Sciences (Basel, Switzerland), 6, E26.

[51] Feske, M.L., Teeter, L.D., Musser, J.M. and Graviss, E.A. (2011) Giving TB Wheels: Public Transportation as a Risk Factor for Tuberculosis Transmission. Tuberculosis, 91, S16-S23. https://doi.org/10.1016/j.tube.2011.10.005

[52] Zhang, Y., Liu, Q.-Y., Luan, R.-S., Liu, X.-B., Zhou, G.-C., Jiang, J.-Y., Li, H.-S. and Li, Z.-F. (2012) Spatial-Temporal Analysis of Malaria and the Effect of Environmental Factors on Its Incidence in Yongcheng, China, 2006-2010. BMC Public Health, 12, 544. https://doi.org/10.1186/1471-2458-12-544

[53] Zhao, F., Cheng, S., He, G., Huang, F., Zhang, H., Xu, B., Murimwa, T.C., Cheng, J., $\mathrm{Hu}, \mathrm{D}$. and Wang, L. (2013) Space-Time Clustering Characteristics of Tuberculosis in China, 2005-2011. PLoS ONE, 8, e83605. 
https://doi.org/10.1371/journal.pone.0083605

[54] Coleman, M., Coleman, M., Mabuza, A.M., Kok, G., Coetzee, M. and Durrheim, D.N. (2009) Using the SaTScan Method to Detect Local Malaria Clusters for Guiding Malaria Control Programmes. Malaria Journal, 8, 68.

https://doi.org/10.1186/1475-2875-8-68

[55] Dietz, N.A., Sherman, R., Mackinnon, J., Fleming, L., Arheart, K.L., Wohler, B. and Lee, D.J. (2011) Toward the Identification of Communities with Increased Tobacco-Associated Cancer Burden: Application of Spatial Modeling Techniques. Journal of Carcinogenesis, 10, 22. https://doi.org/10.4103/1477-3163.85184 